\title{
Characterizing DNA Star-Tile-Based Nanostructures Using a Coarse-Grained Model
}

\author{
John S. Schreck, ${ }^{* \dagger}$ Flavio Romano, ${ }^{\ddagger \dagger}$ Matthew H. Zimmer, ${ }^{\dagger}$ Ard A. Louis, ${ }^{\S}$ and Jonathan P. K. Doye ${ }^{* \dagger}$ \\ ${ }^{\dagger}$ Physical and Theoretical Chemistry Laboratory, Department of Chemistry, University of Oxford, South Parks Road, Oxford OX1 \\ 3QZ, United Kingdom \\ ${ }^{\ddagger}$ Dipartimento di Scienze Molecolari e Nanosistemi, Universitá Ca’ Foscari Venezia, I-30123 Venezia, Italy \\ ${ }^{\S}$ Rudolf Peierls Centre for Theoretical Physics, University of Oxford, 1 Keble Road, Oxford OX1 3NP, United Kingdom
}

Web-Enhanced Feature S Supporting Information

\begin{abstract}
We use oxDNA, a coarse-grained model of DNA at the nucleotide level, to simulate large nanoprisms that are composed of multi-arm star tiles, in which the size of bulge loops that have been incorporated into the tile design is used to control the flexibility of the tiles. The oxDNA model predicts equilibrium structures for several different nanoprism designs that are in excellent agreement with the experimental structures as measured by cryoTEM. In particular we reproduce the chiral twisting of the top and bottom faces of the nanoprisms, as the bulge sizes in these structures are varied due to the greater flexibility of larger bulges. We are also able to follow how the properties of the star tiles evolve as the prisms are assembled. Individual star tiles are very flexible, but their structures become increasingly well-defined and rigid as they are incorporated into larger assemblies. oxDNA also finds that the experimentally observed prisms

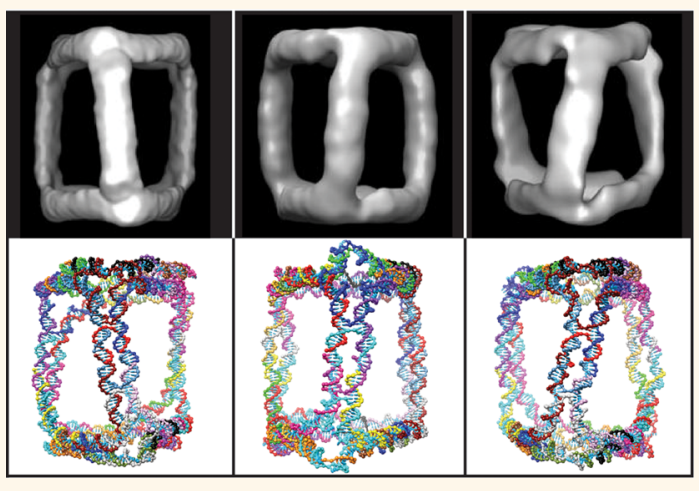
are more stable than their inverted counterparts, but interestingly this preference for the arms of the tiles to bend in a given direction only emerges after they are part of larger assemblies. These results show the potential for oxDNA to provide detailed structural insight as well as to predict the properties of DNA nanostructures and hence to aid rational design in DNA nanotechnology.
\end{abstract}

KEYWORDS: DNA nanotechnology, self-assembly, molecular simulation, DNA star tile, coarse-grained modeling

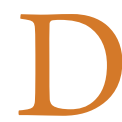
NA has become a leading material of choice for use in creating complex nanoscale structures for potential use in drug delivery, ${ }^{1,2}$ biosensors, ${ }^{3}$ and molecular computation. $^{4}$ In just 30 years since the advent of DNA nanotechnology, ${ }^{5}$ a huge range of different structures can now be made from DNA by using a variety of design and assembly strategies. $^{6-14}$ New software also makes designing large nanostructures with tailored properties easier than ever. ${ }^{15,16}$ Some of these synthetic structures are static and include crystals, ${ }^{17-19}$ polyhedra, ${ }^{12,20-25}$ wire-frame designs, ${ }^{11,26,27}$ and topological structures such as mobius strips, ${ }^{28}$ while others are "active" systems that include walkers, ${ }^{29,30}$ gears and hinges, ${ }^{31}$ robots, ${ }^{32,33}$ and crank-sliders. ${ }^{34}$ However, having total control over the structural as well as time-dependent properties of selfassembled DNA nanostructures remains a significant design challenge. In working toward this goal considerable progress has been made. For example, the relative flexibility of components in systems made from DNA duplexes and single strands can be exploited to create complex structures. ${ }^{12,25,35}$

One such strategy for building $2 \mathrm{D}$ and $3 \mathrm{D}$ structures is tilebased assembly, in which the flexibility of the assembly units (e.g., the tiles) can often be controlled by making simple changes to the design sequences. ${ }^{36,37}$ There are many different types of tiles for use in creating nanostructures. ${ }^{9,10,12,38-41}$ Some tiles are rigid, while others possess more flexibility. For example, the persistence length of double crossover (DX) tiles, which are structures containing two helices connected together by two crossover junctions, is about twice that of ordinary DNA helices. ${ }^{42}$ Multi-arm star tiles, which are structures that are in some ways like multiple DX tiles connected together by a circular strand, can have their flexibility tuned by varying the sizes of the bulges that are present at the center of the tile.

Figure 1a shows a three-arm tile as represented by the oxDNA model. Experiments have found that when the bulge size is small (one or two nucleotides), tile motifs may assemble into $2 \mathrm{D}$ arrays, ${ }^{40}$ whereas when larger bulges are incorporated into tile designs, the tiles may assemble into $3 \mathrm{D}$ polyhedra as the bulges significantly increase the amount of flexibility. ${ }^{23}$

Received: December 5, 2015

Accepted: March 24, 2016

Published: March 24, 2016 


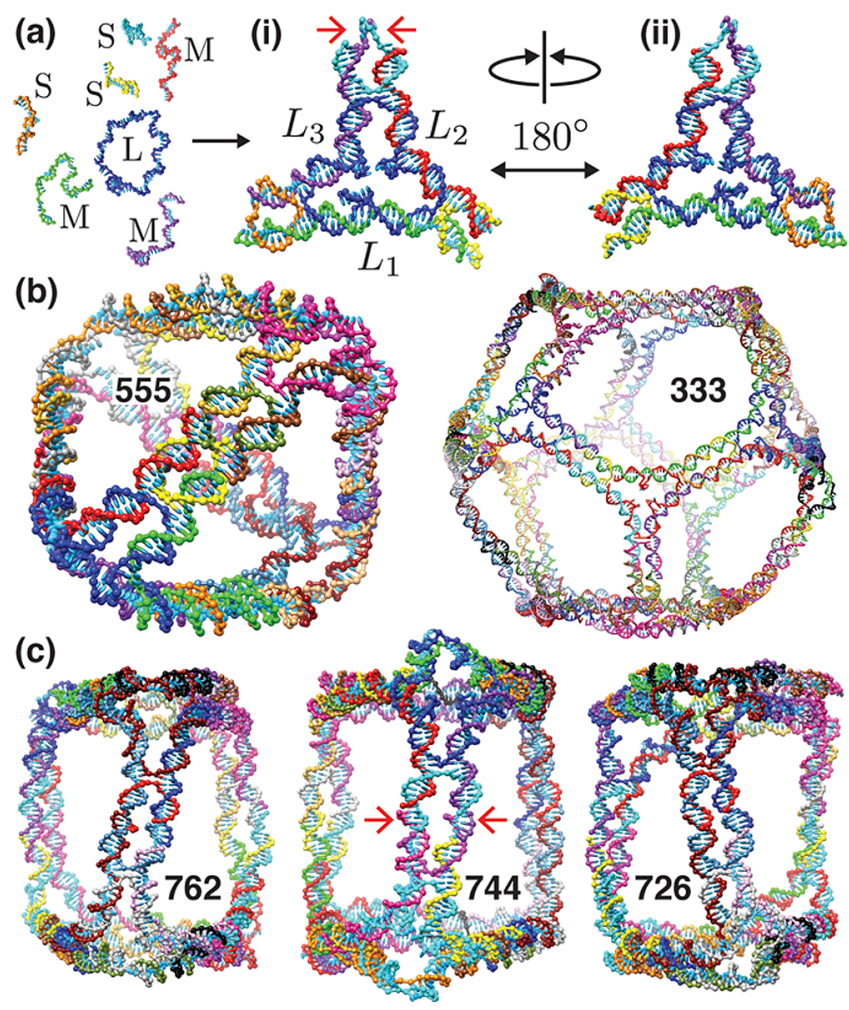

Figure 1. (a) Three-arm star tiles are composed of one long (L) circular strand (dark blue), three medium-sized (M) strands of equal length (green, purple, red), and three short (S) strands of equal length (orange, yellow, cyan). Two viewpoints of a star-tile configuration are shown that illustrate its (i) front and (ii) back face. $L_{i}$ denotes the number of nucleotides in the bulge region. (b) Examples of symmetric star-tile assemblies include tetrahedra $\left(L_{1} L_{2} L_{3}=555\right)$ and dodecahedra (333), which contain four and 20 identical tiles, respectively. (c) Examples of nanoprism assemblies composed of asymmetric star tiles (from left to right: 762, 744, 726). Each nanoprism contains six identical tiles and exhibits a chiral twist between the top and bottom faces. Red arrows in (a,ii) and $(c)$ indicate an example of where the tiles join together at their sticky ends to form an edge of the polyhedron.

Structures assembled from identical tiles that have been experimentally characterized include tetrahedra, ${ }^{22}$ cubes, ${ }^{24}$ octahedra, ${ }^{43}$ dodecahedra, ${ }^{22}$ icosahedra, ${ }^{23}$ buckyballs, ${ }^{22}$ and nanoprisms. ${ }^{44}$ Bulge size, the number of arms, the length of the arms, and the sequence of each arm's single-stranded sticky ends are used to control the product. The use of multiple tile types can further increase the range and complexity of the polyhedra formed. ${ }^{45-47}$ A tetrahedron and a dodecahedron are illustrated in Figure 1b. Similar structures have also been recently achieved using an "origami"-like approach (i.e., with scaffold and staple strands rather than tiles) that again uses bulges to control the flexibility at the vertices in these structures. ${ }^{27}$ By contrast, for DNA origami "tripod" tiles (each tile is a full-size origami), which have been used to produce a range of very large polyhedra, assembly only occurs when struts are incorporated into the designs. These struts stiffen the tiles and give them well-defined interarm angles that specify the target polyhedron.

The size of the bulges in the star tiles can also be used to fine-tune the structure of the target polyhedron. For example, Zhang et al. created a series of triangular nanoprisms with different degrees of chiral twist between the top and bottom faces (illustrated in Figure 1c) by adjusting the individual bulge sizes in the three-arm tiles. ${ }^{44}$ Based on the experimentally observed products for different designs, it has been conjectured that the arms in star tiles may possess a slight tendency to bend in a preferential direction. ${ }^{40,43,48}$ This would help explain, for example, why choosing adjacent tiles to face in the opposite direction from each other leads to $2 \mathrm{D}$ structures being formed over 3D structures, because the inherent curvature effects would cancel out. ${ }^{40,49}$ Similarly, when the tiles are designed to face the same direction in assemblies, they are observed to close up into 3D polyhedra, with the hypothesis being that the cumulative curvature aids closure. ${ }^{22}$

Detailed analysis of cryogenic transmission electron microscopy (cryoTEM) structures of octahedra that form from fourarm tiles has shown the arms preferentially bend in one direction in the assembled structure. ${ }^{43}$ Similarly, the sense of the twist in the chiral nanoprisms is also consistent with this direction of curvature. Figure 1a illustrates the two faces of a three-star tile, which can be distinguished from each other by comparing the major and minor groove patterns of the doublehelix sections within each tile. We label the two faces the "front" and "back" face, where the groove pattern of the front face is equivalent to that which points outward in the octahedron.

Molecular simulations have the potential to provide insight into the structure, flexibility, and curvature effects in DNA tiles and their assemblies. In order to describe these structures, a model needs to incorporate the relevant physics that is capable of describing DNA at similar environmental conditions as used in the experiments. Second, a model needs to be able to simulate time and length scales that are comparable to those accessed in experiments, which may be difficult when simulating large structures. Large DNA origamis and nanocages have already been simulated using all-atom models, ${ }^{50-53}$ however they are too computationally expensive to routinely equilibrate such structures. Coarse-grained models, although providing less molecular detail, have the potential to achieve these aims.

The oxDNA coarse-grained model has proven particularly powerful for understanding basic DNA physics in a variety of systems, ${ }^{54-57}$ and it is increasingly being used to study nanotechnological systems. ${ }^{48,58}$ The degree of coarse-graining in the model, which is at the nucleotide level, captures enough of the details of DNA that are relevant for describing DNA nanostructures including duplex stability, the relative flexibility in single and double-stranded DNA, and the flexibility changes induced in duplexes by bulge loops. ${ }^{48,59-62}$ When the model is combined with the computational power of graphical processing units (GPUs), it becomes possible to comfortably simulate large systems that may contain thousands of nucleotides at a microscopic level of detail and for long enough to generate ensembles of configurations representative of the equilibrium structure.

In this article, we use oxDNA to model the structural properties of the three-arm star tiles of ref 44 and the twisted nanoprisms that they form. We compare our results with reconstructions of cryoTEM images of the real structures and monitor how the flexibility and curvature of the tiles evolve as the prism grows. The results presented here should provide both a test of oxDNA's predictive power for modeling DNA nanostructures and detailed structural insights that will aid the rational design of nanostructures that use bulges as a way to control flexibility and therefore global structure. 

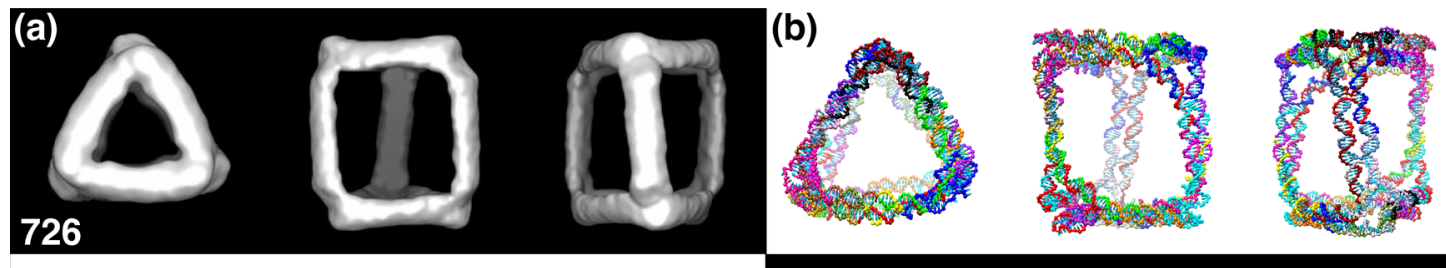

(c)
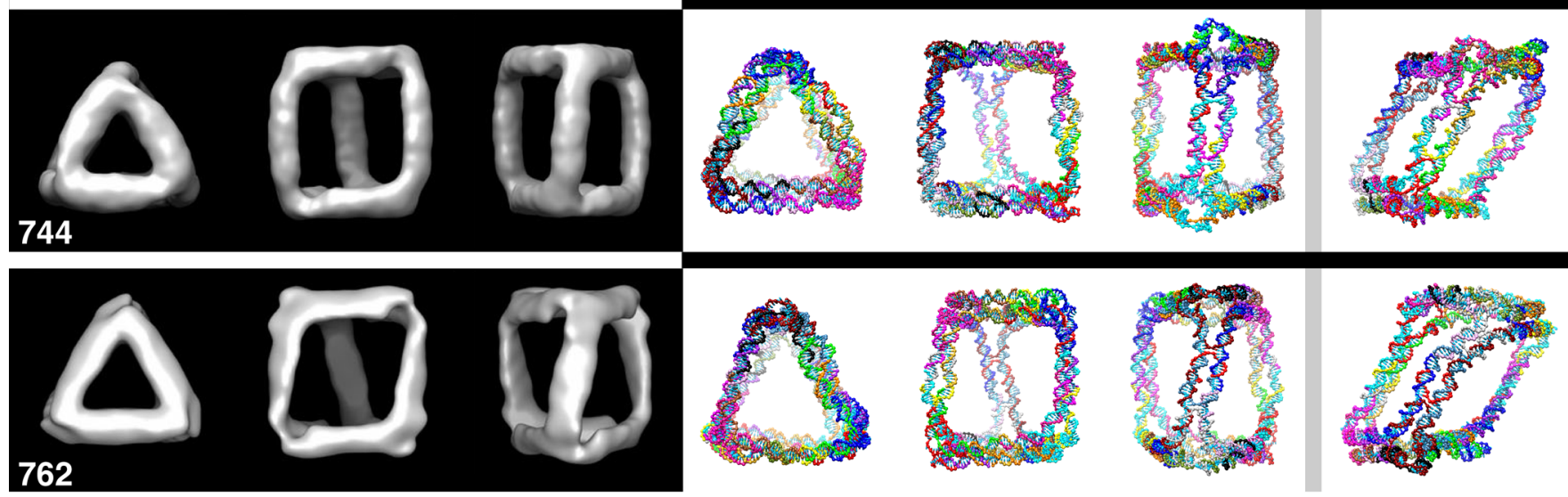

Figure 2. (a) Reconstructed 3D maps of prisms obtained from cryoTEM imaging. ${ }^{44}$ (b) Typical oxDNA configurations for prisms. Top row: 726 prisms, middle row: 744 prisms, bottom row: 762 prisms. In (b), oxDNA configurations with a twist angle that is approximately equal to the mean twist angle for a prism type are shown, while those shown in (c) are rarer examples of prisms that have become strongly sheared and which clearly illustrate the effect that shearing may have on prism geometry. Images in (a) have been modified and reproduced with permission from ref 44 . Copyright 2012 John Wiley \& Sons, Inc.

\section{RESULTS AND DISCUSSION}

Experimental Systems and Model. Star-Tile Systems. Three-arm star tiles are assemblies containing one "long" (L) circular strand containing 78 nucleotides, three "medium" (M) strands each containing 42 nucleotides, and three "short" (S) strands each containing 21 nucleotides. These different strands are illustrated along with a fully assembled three-arm tile in Figure 1a. During assembly, sections of the three M strands hybridize with the $\mathrm{L}$ strand to form three duplexes that contain bulges, where the bulges are at the center of the tile to allow flexible movement of the arms. At the terminal ends of each tile arm are two single-stranded "sticky" sequences typically four bases in length that are complementary and connect tiles together when assembled. The number of nucleotides in a bulge region is denoted $L_{i}$, thus each three-arm tile, and the prism composed of identical copies of this tile, can be described with the notation: $L_{1} L_{2} L_{3}$, as is illustrated in Figure 1.

A three-arm tile possesses 3 -fold rotational symmetry when the $M$ and $S$ strands are identical and the circular $L$ strand is composed of three repeating sequences. In experiments it was found that symmetric three-arm tiles can assemble to form tetrahedra when the bulge size in the $\mathrm{L}$ strand is 5 , and dodecahedra and buckyballs when the bulge size is 3, depending on the concentration of monomer tiles, with higher concentration yielding the buckyballs. ${ }^{22}$

The three-arm tiles that we consider here are asymmetric both in the bulge sizes in the L strand and the sticky ends of the $S$ strands and were originally designed to form triangular prisms. ${ }^{44}$ The edges of the fully assembled prisms are 42 base pairs long (i.e., four helical turns), which causes the tiles to face in the same direction in the final assemblies, and may promote the formation of closed polyhedra during assembly due to tile curvature effects. The basic triangular prism design involves $L_{1} L_{2} L_{3}=744$ tiles, where the $L_{1}$ bulges are at the corners of the triangular faces and the $L_{2}$ and $L_{3}$ bulges at the corners of the approximately square faces. That triangular prisms formed in the experiments suggests that the flexibility provided by the $L_{1}$ $=7$ bulges promotes the formation of triangular prisms, as opposed to, for example, square or pentagonal prisms. When $L_{2}$ and $L_{3}$ are not the same, the bulged duplexes at the corners of the quadrilateral faces of the prisms will possess different degrees of flexibility, thus giving rise to twisted chiral prism shapes. The 753 and 735 prisms were designed to twist in opposite directions with respect to the 744 prism, with smaller and larger bulges correlating with smaller and larger bend angles possible in the quadrilateral faces, respectively. The 762 and 726 prisms were observed to behave similarly, but with a larger magnitude for the chiral twist. ${ }^{44}$

Coarse-Grained Model for DNA. In oxDNA, a single strand of DNA is modeled as a chain of rigid nucleotides. Interactions contributing to the potential energy of a particular configuration include stacking, cross-stacking, coaxial stacking, hydrogen bonding, excluded volume, and backbone chain connectivity. Base-pairing interactions obey Watson-Crick specificity (i.e., A-T or G-C pairs), but other interactions such as Hoogsteen pairs are excluded from the model. Details of the interactions contributing to the oxDNA potential can be found elsewhere, ${ }^{48,59}$ and the simulation code for oxDNA can be downloaded from the oxDNA Web site. ${ }^{63}$ For the current study we use the latest version of the model which includes different groove widths for the helix (i.e., major-minor grooving) and fine-tuned structural properties to improve modeling of DNA origami as well as the relative flexibility of adjacent TT bases in a sequence, all of which will be important for studying tiles and nanocages. ${ }^{48}$

Global Structure. To calculate average properties for both tiles and prisms, we ran long dynamics simulations while periodically saving configurations for analysis. Animations of short trajectories for the 744 tile and the 744 prism are included in Movies 1, 2, 3, and 4 (see Supporting Information for 
additional explanation). Further details regarding the simulations are discussed in the Methods section. In Figure 2, we compare the single-particle $3 \mathrm{D}$ reconstructed maps of DNA prisms obtained from cryoTEM images in ref 44 with representative oxDNA configurations for the 762, 744, and 726 prisms. The chiral twist of these configurations has a twist value near the mean value for its computed twist angle distribution $p(\omega)$. The comparison between Figure $2 \mathrm{a}$ and $\mathrm{b}$ clearly shows that the model predicts equilibrium prism structures for a variety of designs that are very similar to the experimentally determined $3 \mathrm{D}$ maps. In particular, our structures reproduce very well the chiral twist seen experimentally, which is driven by the tendency of bulged duplexes with smaller bulge loops to bend less. Both the $3 \mathrm{D}$ maps and the oxDNA configurations show that the top and bottom faces are roughly equilateral triangles and are roughly perpendicular to the sides of the prisms. The quadrilateral faces of the 744 prism are close to being square in shape. Prisms containing bulges of sizes 2 and 3 more clearly have faces possessing parallelogram shapes. The struts connecting the vertices together, which are made of two parallel linked double helices, are also roughly straight.

The twist angle distributions $p(\omega)$ for different prism types are shown in Figure 3. We calculated the twist of a prism

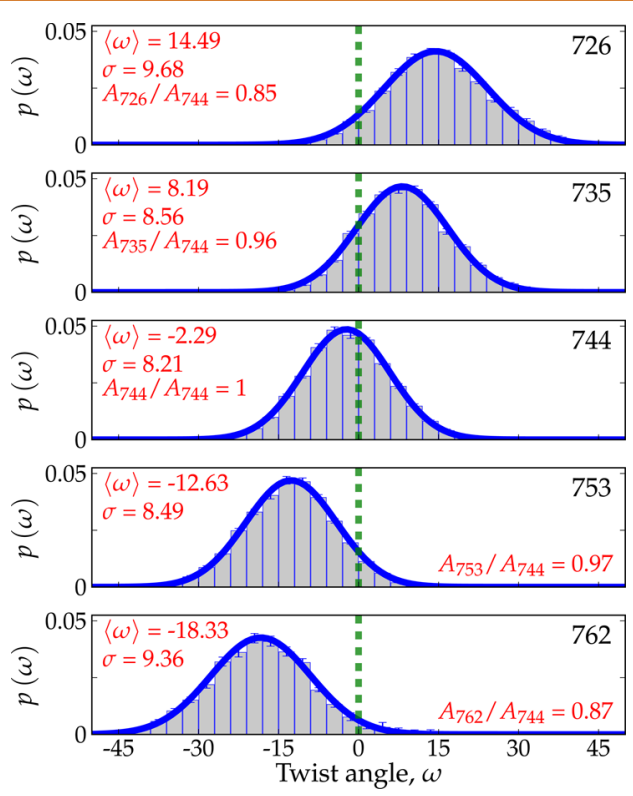

Figure 3. Probability distributions for the twist angle $\omega$ between the top and bottom triangular faces are shown for the five nanoprisms simulated. Solid blue lines are Gaussian fits to the raw data obtained from oxDNA. Dashed green lines in each plot serve as a reference for $0^{\circ}$ of twist. Also listed in each plot is the mean twist value $\langle\omega\rangle$, the standard deviation $\sigma$, and the relative amplitude $A_{k} /$ $A_{744}$, for each of the Gaussian distributions.

configuration by using a simple scheme which measures the angular difference between the top and bottom triangular faces of a prism (see Figures S1 and S2 in the Supporting Information for more details). The prisms shown in Figure $2 \mathrm{~b}$ were selected from a set of configurations which all had a twist angle within $2^{\circ}$ of the mean twist value for a particular prism type. We take $735 / 726$ and $753 / 762$ prisms to have clockwise (positive) and counterclockwise (negative) twists, respectively.
Overall, oxDNA predicts that the twist angle distributions for prisms 735 (726) and $753(762)$ are approximately symmetrical with respect to the distribution for the 744 structure. However, the $3 \mathrm{D}$ maps clearly show that the 744 prism is slightly twisted in the same counterclockwise direction as the 762 prism. The finite twist in the 744 structure is also predicted by oxDNA, as is clear in Figure 3, where we have measured a mean twist of $-2.3^{\circ}$. The 735 and 753 prisms each have mean values approximately $+10^{\circ}$ and $-10^{\circ}$ with respect to the mean value of the 744 prism, respectively, while the corresponding values for the 726 and 762 prisms are $+16^{\circ}$ and $-16^{\circ}$. Figure 3 also shows that the twist angle distribution is sharpest for the 744 pyramid and becomes slightly wider as the tiles become more asymmetric. The breadth of these distributions means that some fraction of the time the prisms will adopt a chiral twist that is opposite to that of the average structure. For example, about $16 \%$ and $7 \%$ of the configurations for the 735 and the 726 prisms, respectively, exhibit twist in a counterclockwise direction.

In addition to possessing a relatively broad degree of chiral twist, the triangular faces of the prisms may also be "sheared" with respect to each other. Example configurations showing a high degree of shear are illustrated in Figure $2 c$. To quantify the degree of shear we use a simple scheme where we project the centers of mass of the top and bottom faces of the prism onto a plane defined using vectors normal to the triangular faces and then measure the distance between these points on that plane (for more details see Figure S2 and Figure S3 in the Supporting Information). Most prisms are not as strongly sheared as those shown in Figure 2c. However, a moderate degree of shearing is reasonably common (see Figure S3 in the Supporting Information) and is a significant deformation mode of the prism away from its mean structure, further highlighting the degree of flexibility still present in the tiles even when part of a complete prism. The fluctuations in the twist and shear of the prisms are also evident in the animation of the 744 prism in Movies 1, 2, 3, and 4 (see Supporting Information for additional explanation and Figure S7).

Local Structure. In order to characterize the relative flexibility at the vertices in the prisms, we focus on the bulged duplexes within the star tiles, which are illustrated schematically in Figure 4a. We measure the flexibility of these motifs as characterized by a bend angle $\theta$ between the duplex sections that come together at the bulge and the relative torsional twist $\phi-\psi$ between these sections, each of which have a twisting degree of freedom around its helical axis. These quantities are illustrated in Figure $4 \mathrm{~b}$ for a duplex with a bulge and in Figure $4 \mathrm{c}$ for the bulged duplexes in a star tile. Details regarding the computation of the bend and torsional twist angles are given in the Supporting Information.

Bulged Duplex Structure Classes. We classify the bulged duplexes in the tiles into two main structural classes, namely "stacked" and "unstacked" depending on whether the stack opposite the bulge (cyan circles in Figure 4b) is intact or broken, respectively. The details of the criterion used to define these states are given in the Supporting Information. Previous investigations of bulged duplexes with oxDNA have identified two subclasses of stacked configurations: ${ }^{62}$ (a) the stack across the bulge remains intact (red in Figure 4), the two duplex arms meeting at the bulge are roughly straight, and the twist between the two duplex sections is approximately equal to the rise per base pair in the oxDNA model, which is roughly $32^{\circ}$; (b) the stack across the gap is broken and some of the bases from the 


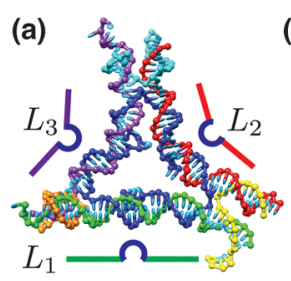

(b)
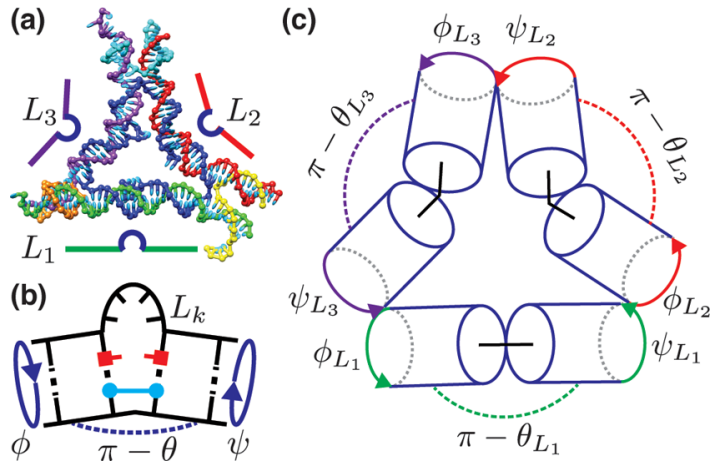

Figure 4. (a) The locations of the bulged duplexes in a single star tile are illustrated with the sizes of the bulges denoted $L_{1}, L_{2}$, and $L_{3}$. (b) A schematic representation of the bulge region within a bulged duplex. The stack between bases (circles) opposite the bulge is represented in cyan and is drawn intact, whereas the coaxial stack between bases (squares) that are adjacent to the bulge (red) is drawn as broken. The duplex arms meeting at the bulge are bent at an angle $\theta$ and have a torsional twist with respect to one another of angle $\phi-\psi$, where $\phi$ and $\psi$ are the twist angles for each duplex section. (c) Bulged duplexes in star tiles are represented with cylinders. Bend and torsional twist angles for each bulged duplex are denoted in the illustration. In (b) and (c) we use the same convention for the bend angle as used in ref 62 .

bulge loop may be inserted into the duplex. Base insertion causes the two duplex sections to bend slightly away from the bulge, and the relative twist at the gap is increased due to the extra bases. For the unstacked configurations where the stack opposite to the bulge is broken, the bulge acts somewhat like a hinge between the two duplex sections. Thus, the duplex can be strongly bent with a maximum angle determined by the bulge size. The degree of twisting also markedly increases in unstacked configurations.

In Figure 5, we illustrate and analyze these structural classes in configurations of star tiles and prisms. These snapshots are complemented by the animations of the 744 tile and prism provided in the web-enhanced content, and the analyses of the trajectories in terms of the stacking states of the bulged duplexes, and the bend angles in Figures S5 and S6 in the Supporting Information. In particular, these show that the bulged duplexes fluctuate between stacked and unstacked states on nanosecond time scales.

For example, Figure 5a illustrates a 744 configuration where all three bulged duplexes are stacked with a small static bend at the two bulges that have bases inserted into the gap and a roughly straight duplex at the other bulge. Instead, the bending required by the overall tile geometry is spread out through the duplexes. This form most resembles the idealized schematics of the tile with a roughly flat structure and the two duplexes at the ends of each arm not far from parallel. By contrast, Figure $5 b$ shows a configuration for the 744 tile where all of the stacks opposite to the bulge regions are broken, and the overall structure is much less well-defined with different bend and twist angles at each bulge. This unstacking allows the tiles to adopt a wide range of structures facilitated by the relative freedom in the orientations of the duplex sections on either side of the bulges when in an unstacked state. In particular, the two helices that pass through the four-way junctions in each arm have more freedom to adopt the twisted "X-like" configuration that is favored in isolated four-way junctions. ${ }^{64}$

Similar characteristics are evident in the configurations in Figure $5 \mathrm{c}$ and $\mathrm{d}$ which shows tiles possessing different combinations of stacked and unstacked bulged duplexes where the unstacking again allows the tile to exhibit greater flexibility. Overall, Figure 5a-d clearly shows that the individual tiles can adopt a diverse of range of structures facilitated by the flexibility provided by the bulged duplexes in the tiles, and this conclusion is further confirmed by the animation of a 744 tile in the web-enhanced content.

Figure $5 \mathrm{e}-\mathrm{h}$ illustrates these classes in complete prisms. The tiles now have a much more well-defined structure as the helices in each arm are now constrained to be parallel by the intertile bonding. The remaining freedom is mainly in the angles between the arms, but the global structure of the prism can play a significant role in further constraining the flexibility of the bulged duplexes. For example, those in the triangular
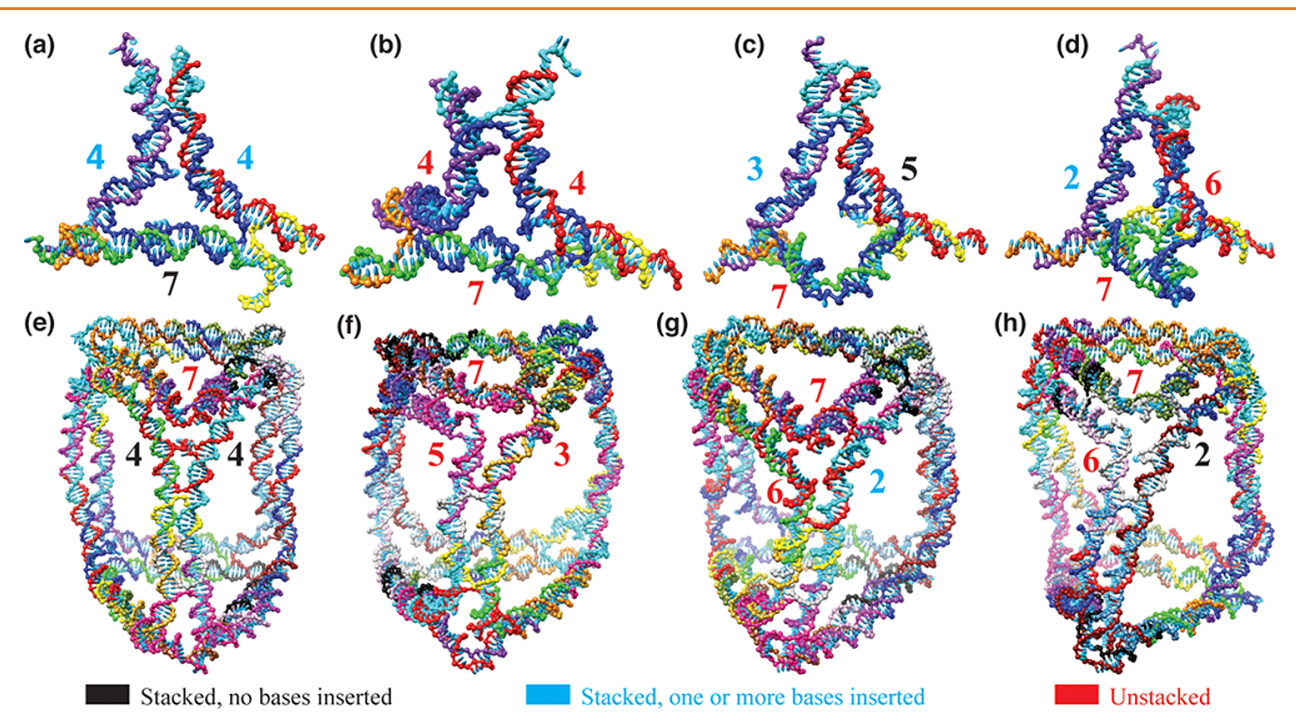

Figure 5. Top panels show different conformations of free star tiles with varying bulge size: (a) and (b) 744, (c) 753, (d) 762. The bottom panels show different conformations of prisms: (e) 744, (f) 753, (g) and (h) 762 . The bulge size of each bulged duplex is indicated in the panels with the color of the number indicating stacked bulged duplexes with no inserted bases (black) or inserted bases (cyan) or unstacked bulged duplexes (red). 
faces are always unstacked and bent at large bending angles (Figure $5 \mathrm{e}-\mathrm{h}$ ). By contrast, the bulged duplexes at the vertices of the quadrilateral faces can be stacked or unstacked with a wider variety of possible bend angles. When unstacked the bending is mostly localized at the bulge, whereas when stacked, the bending is distributed along the duplex as is evident in the 744 configuration in Figure 5e, for which both 4-nucleotide bulged duplexes are stacked.

Figure $5 \mathrm{~g}$ and h shows configurations for 762 prisms which have chiral twist angles close to the mean value shown in Figure 3. These configurations illustrate the tendency for the smaller bulge to adopt a stacked configuration with a smaller bend angle that in part underlies the tendency of the prism to adopt a twisted structure. However, even when both are unstacked, as is the case in Figure 5f, the prism can still exhibit twist because the shorter bulge somewhat restricts the bend angle that can be achieved.

Average Structure Properties. We can further quantify some of the trends we have identified from inspection of individual configurations by statistically analyzing the geometric properties of the bulged duplexes in the tiles. First, we calculate the probability that bulged duplexes in free tiles and in prisms are stacked or unstacked for different bulge sizes, and the average bend angle as a function of bulge size. These quantities are shown in Figure $6 a$ and $b$, respectively.

Similar to the free bulged duplexes studied using oxDNA in ref 62, smaller bulges of sizes 2 and 3 clearly prefer to adopt a stacked configuration in both tiles and prisms because it is freeenergetically more favorable for them to either flip out the bulge bases or incorporate them into the helix rather than to unstack and stretch out the bulge. Figure 6a shows that as bulge
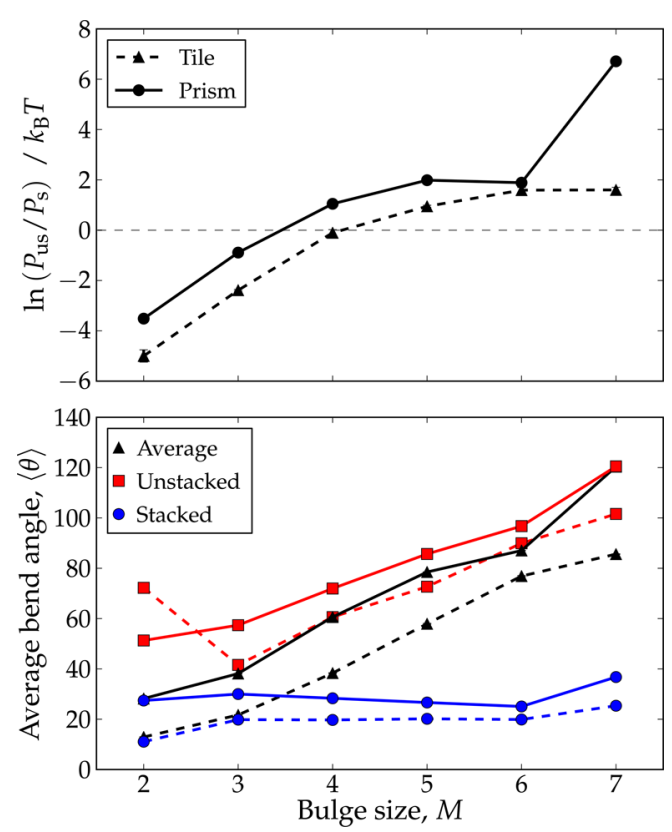

Figure 6. (a) Probability of unstacked-to-stacked configurations of bulged duplexes in free star tiles (dashed lines) and in prisms (solid lines) for the different bulge sizes used in the structures. (b) Average bend angle (black triangles), the average stacked bend angle (blue circles), and the average unstacked bend angle (red squares) for a bulged duplex of varying size are shown for free star tiles (dashed lines) and prisms (solid lines). In prisms, bulge sizes 2-6 are found in quadrilateral faces, while bulges of size 7 are found in triangular faces. size increases, there is an increasing likelihood that the bulged duplex will be unstacked. The likelihood of being unstacked is greater for the bulged duplexes in a tile than individual bulged duplexes, $^{62}$ because the tile structure imposes a bending requirement favoring the unstacked state. Similarly, the bulged duplexes that are in tiles that are part of a complete prism are even more likely to be unstacked because of the additional bending required by the prism geometry. The sharp jump in unstacking for the 7-nucleotide bulges in prisms is because they are at the corners of the triangular rather than quadrilateral faces.

The values for the average bend angles for tiles and prisms, plotted in Figure $6 \mathrm{~b}$, clearly reflect the stacked to unstacked ratio of bulged duplexes. For the smallest bulges, the average bend angle is equal to the that of the stacked configurations, which tends to remain small. For large bulge sizes, the average bend angle tends toward that for the unstacked configurations, which may be strongly bent. For both tiles and prisms, the average bend angle for a stacked configuration is roughly constant, whereas the average bend angle for a configuration and the average bend angle for an unstacked configuration increase as bulge size increases. The latter increase reflects the greater freedom to open up to large bend angles for longer bulges. $^{62}$ It is also clear from Figure $6 \mathrm{~b}$ that the bend angles are slightly larger in the complete prisms than the individual tiles, again due to the geometric requirements on the interedge angles in the prisms.

Distributions for Bending and Torsional Twisting Angles. To get a more complete pictures of the flexibility introduced into the tiles by bulges and the changes in tile flexibility caused by the prism structure, in Figure 7 we compare the distributions for the bend and twist angles defined in Figure $3 \mathrm{c}$ as the bulge size varies. Of course, the tendency for greater unstacking in prisms and the trends in bend angle noted above are again evident, however the distributions also provide a detailed picture of how the angular flexibility of the tiles changes on assembly.

For bulged duplexes in the stacked state, apart from a reduced tendency to bend into the bulge (i.e., negative $\theta$ ) and a small increase in bend angle, both the bend and twist distributions do not change much when tiles are incorporated into prisms, because the continuity of the stacking provides a strong structural constraint. The double peak structure of the twist angle distributions reflects the two possible types of stacked states, namely those that have all the bulge bases flipped out of the helix and have a twist difference similar to that of a base pair step in a duplex (i.e., $\sim 35^{\circ}$ ), and those that have one or two bases inserted and consequently have a larger twist difference.

By contrast, for the bulges adopting an unstacked geometry, the angular distributions change considerably for the prisms, first moving to larger bend and twist angles, and second becoming much narrower and more symmetric about the mean. These changes reflect the angular restrictions that are imposed and the flexibility that is lost when the tile arms become fixed into place within the prism structure. The distributions are also significantly narrower for the 7-nucleotide bulges at the vertices of the triangular faces.

Characterization of Tile Curvature. Lastly, we characterize the intrinsic curvature of the three-arm star tile. As noted earlier, in experiments involving four-arm tiles, the arms are observed to bend away from the front face of a tile when assembled into octahedra. ${ }^{43}$ The observed chirality of the 

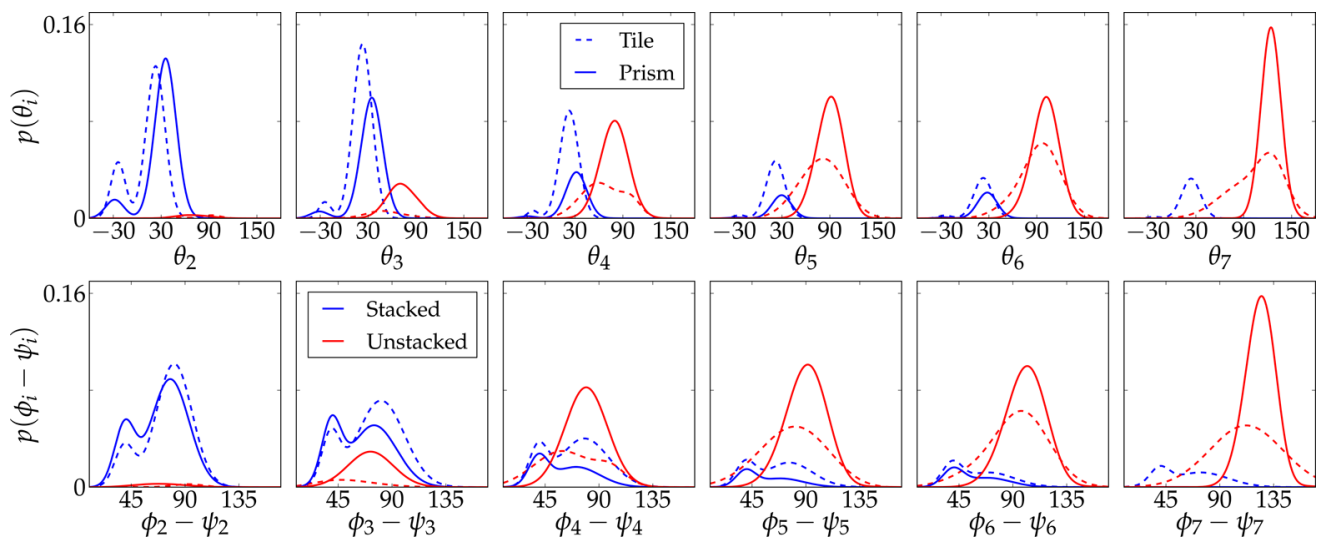

Figure 7. Probability distributions for the bend angle $\theta$ (top panels) and the torsional twist angle $\phi-\psi$ (bottom panels) for bulges of different size. In all figures, solid and dashed lines refer to bulged duplexes in prism and free tiles, respectively, while blue and red colors refer to stacked and unstacked populations, respectively. The label $i$ in, for example, $p\left(\theta_{i}\right)$ refers to the bulge size.

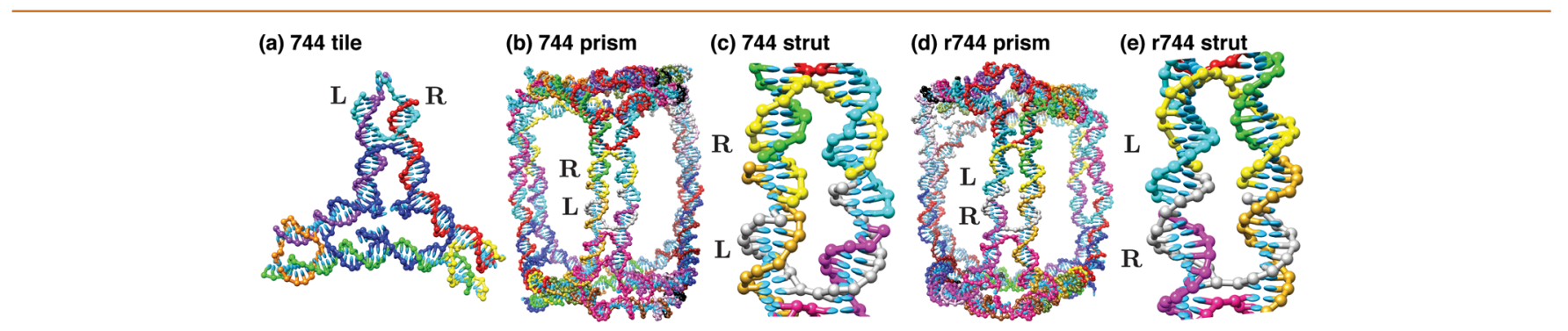

Figure 8. (a) 744 tile configuration with double-helix sections L (cyan-purple helix) and R (cyan-red helix) highlighted for one arm. (b) Experimentally observed 744 prism with the front face of tiles pointing away from the prism. (c) Close-up view of a strut for the 744 prism. (d) Inverted counterpart to the 744 prism where the back face of the tiles points away from the prism. (e) Close-up view of a strut in the $\mathrm{r} 744$ prism.

prisms considered here also implies the same direction of curvature, as the inverted forms of the prisms would have the opposite twist. The inverted prism forms are the prisms that would result if the tile had the opposite curvature, that is, the back faces of the star tiles all would point outward from the structure. We denote the inverted form of the 744 prism as "r744". Figure $8 \mathrm{~b}$ shows the prism when the front faces of the tiles all point outward as well as its inverted counterpart in Figure $8 \mathrm{~d}$. Additionally, Figure 8 highlights the major-minor groove patterns of the struts for the two types of prisms (which can be used to identify the prism types). We have previously shown that the latest version of the oxDNA potential predicts that the remaining free arms of three-arm tiles incorporated into triangular-shaped trimers also have a clear preference for pointing away from the front face of the tile, with the incorporation of different groove widths (e.g., major-minor grooving) being key to reproducing this experimentally observed preference. $^{48}$

Bend Angle Distributions for Tile Curvature. To test for this preference in free tiles, we constructed a simple way to measure an angle $\chi$ between the helices in the tile arms and a tile normal vector, $\mathbf{n}$, that is detailed in the Supporting Information. Figure 9a,i illustrates the tile normal vector, two helices within an arm (denoted $\mathrm{L}$ and $\mathbf{R}$ in the figure), and the bend angle $\chi$. Figure $9 \mathrm{a}, \mathrm{i}-$ iii also shows example configurations of the tile bending in different directions. In our measurements, angles are $>90^{\circ}$ if the arms bend away from the front face and $<90^{\circ}$ for bending away from the back face.

Figure $9 \mathrm{~b}-\mathrm{e}$ shows distributions $p(\chi)$ for the free 744 tile, dimers, and trimers made from 744 tiles and the experimentally observed form of the 744 prism in which the arms in all tiles point away from their front faces. The distribution for this prism structure serves as a reference to compare with the other structures. The distribution for this form of the prism is symmetric, sharply peaked, and centered at $124^{\circ}$. Although this angle is clearly considerably constrained by the overall structure of the prism, there is still significant variation $\left(\sigma=17^{\circ}\right)$ due to the thermal fluctuations in the local and global structure of the prism.

Figure $9 \mathrm{~b}$ shows that monomeric free tiles have a much broader distribution than those incorporated into prisms, showing that the helices in the arms of the free tiles are able to fluctuate considerably in their orientation. The distribution is also clearly not symmetric, with a maximum value at around $100^{\circ}$. However, the mean bend angle for the arms is roughly $90^{\circ}$, indicating that a typical tile configuration is relatively flat (Figure 9a,ii) and that as monomers the tiles exhibit no intrinsic curvature. One contributing factor to the width of the distribution is that the two helices in an arm have somewhat different orientational preferences with the L helix in Figure 9a preferring to bend more toward the front face, while the $\mathrm{R}$ helix prefers to bend in the opposite direction. This reflects the tendency of the two helices in the stacked conformation of a free four-way junction to exhibit a chiral twist with respect to each other. In the monomeric tiles, there is sufficient flexibility in the structures for the $\mathrm{L}$ and $\mathrm{R}$ helices to exhibit substantial differences in their $\chi$ values $\left(\sim 40^{\circ}\right)$, but as the arms of the tiles join together to form edges of the prism, the two helices are constrained to be virtually parallel, and this source of variation in $\chi$ is dramatically reduced. Note though that for oxDNA the 
(a) (i)

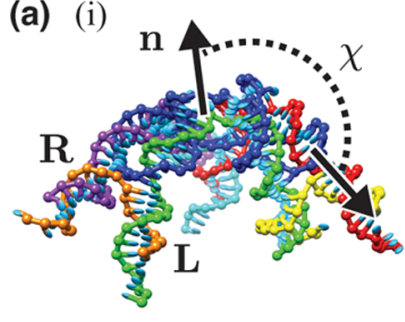

(ii)

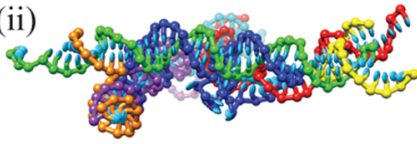

(iii)

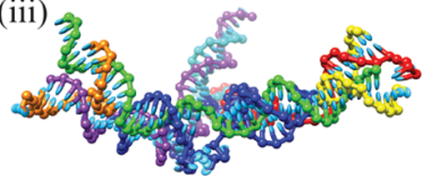

(b)

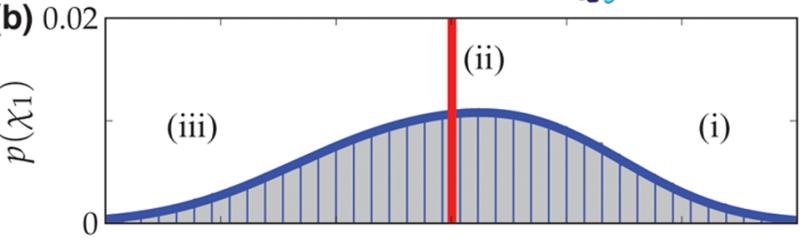

(c)
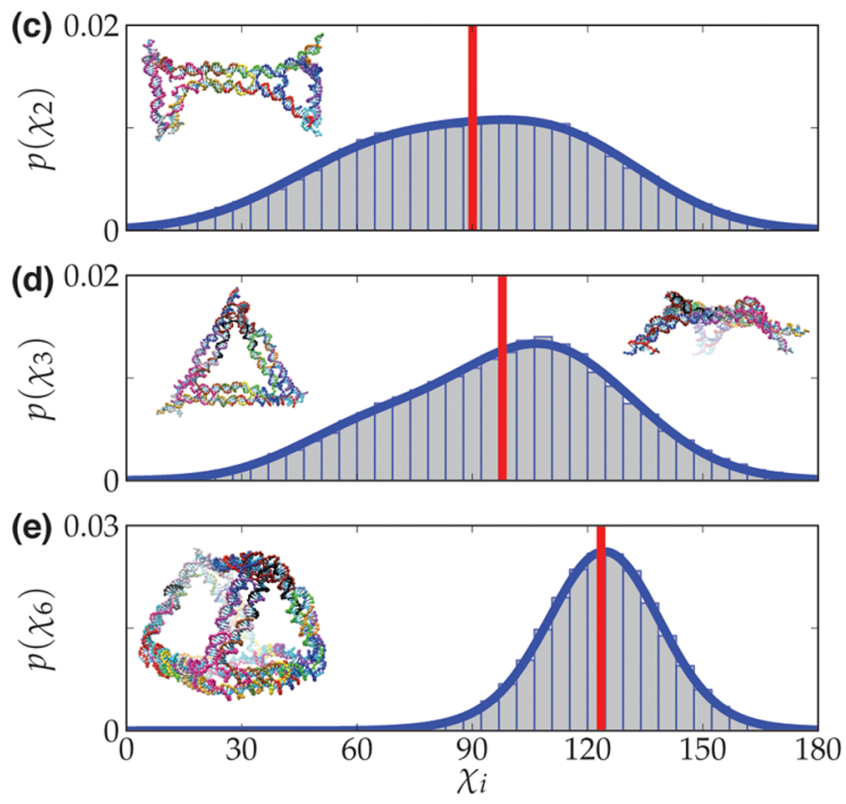

Figure 9. (a) Configurations for a 744 tile illustrating the range of the tile bend angle $\chi$. (i) A tile with all the arms bent with angles $\chi$ $>90^{\circ}$; (ii) a roughly flat tile with $\chi$ approximately equal to $90^{\circ}$ for the three arms; and (iii) a tile bent opposite to that in (i) where $\chi$ is $<90^{\circ}$ for the three arms. Like in Figure 8, the labels L (greenorange) and $\mathrm{R}$ (purple-orange) denote the two helices at the end of a tile arm. From top to bottom, the histograms show the range of angle $\chi$ in (b) monomers, (c) dimers, (d) trimers, and (e) prisms. The $i$ in probability distributions, $p\left(\chi_{i}\right)$, refers to the number of tiles in the assembly. In each plot a vertical red line indicates the average value of the measured curvature.

helices in a free four-way junction prefer to have a left-handed twist, while experiments suggest that real DNA prefers a righthanded twist. ${ }^{64}$

Although one of the three arms in each tile of the dimer is now more structurally well-defined due to the formation of a prism edge (Figure 9c), the $p(\chi)$ distribution for the dimer is still very similar to that for the monomer and, in particular, again shows no preference for a particular curvature; the dimer is on average flat. For the trimer with two of the three arms of each tile now constrained in an edge of the triangle, the $p(\chi)$ distribution begins to narrow somewhat. More interestingly, the trimer shows a significant shift toward bend angles $>90^{\circ}$. The peak in this distribution occurs near $110^{\circ}$, while the average value is approximately $98^{\circ}$.
Our analyses of both the bending at the bulges and the tile curvature show that once a tile has been incorporated into a larger assembly, it loses considerable flexibility. That an intrinsic curvature to the tiles only emerges as they assemble into larger structures is more subtle and must be due, in some way, to a tightening of the coupling between the arms as they become both more rigid and more orientationally constrained by the completion of polygonal faces of the target polyhedron. The resulting preference for the experimentally observed 744 prism (Figure 10a) over its inverted counterpart "r744" (Figure 10b) is clear from the potential energies of the two structures in the oxDNA model. The experimentally observed prism is lower in potential energy by $\sim 56 k_{\mathrm{B}} T$ at a temperature of $20{ }^{\circ} \mathrm{C}$.

Potential Energy Considerations. To get further insight into this preference, we compare the contributions from different terms in the oxDNA potential for the two forms of the 744 prism in Table 1 and in Figure S8. Clearly the stacking is the dominant contributor to the energetic preference for the experimentally observed structure with an approximately 65 $k_{\mathrm{B}} T$ difference between the two forms, although this is partly offset by a higher coaxial-stacking energy (about $16 k_{\mathrm{B}} T$ ).

To identify from where in the prisms this difference in energy arises, we computed the relative energies of each nucleotide in a tile for the two forms, considering each type of interaction in turn. The results in Figure 10 clearly show that the dominant contribution to the lower stacking energy of the preferred form comes from the nucleotides in the three bulges in the L strand. Given the importances of the bulges, we also computed the probability that a bulge adopts a stacked configuration for the two forms. First, we consider the 7nucleotide bulge which is invariably unstacked in both cases. For this bulge, as well as the better stacking energy in the 744 prism, the bases on either side of the bulge are also able to better hydrogen bond to each other. Thus, not only does the detailed geometry of the bulge in the observed form allow better stacking in the bulge, it does so while imposing less stress on the surrounding structure.

Due to the smaller angle imposed on the bulges at the corners of the square faces, both stacked and unstacked configurations are observed for the 4-nucleotide bulges. However, the bulged duplexes in the inverted prism now have a significantly higher probability of being in a stacked configuration ( $57 \%$ for $\mathrm{r} 744$ compared to $35 \%$ for 744 , see Figure S9 and Figure S10 in the Supporting Information), presumably due to greater stress in the unstacked configurations for this form already noted for the 7-nucleotide bulges. Consequently, the stacking energy even more favors the observed form for the 4-nucleotide bulges, because of the additional loss of stacking at the junction between the duplexes and the bulge. But this shift away from the unstacked configuration does lead to a reduction in stress in the nearby duplex sections, and so the hydrogen-bond energy of the base pairs next to the bulge is now lower for the inverted form and there is better coaxial stacking at the nearby four-way junctions.

However, even though we know that including the asymmetry of the helix associated with the major and minor grooves is vital to reproducing the preference for the observed form, ${ }^{48}$ and having narrowed down both which parts of the structure and which energy terms favor the observed form, the structural complexity of these prisms means that it has still not been possible to pinpoint the precise geometric reasons for this preference. Although slightly disappointing, in some ways, this provides further justification for using models such as oxDNA. 
(a)

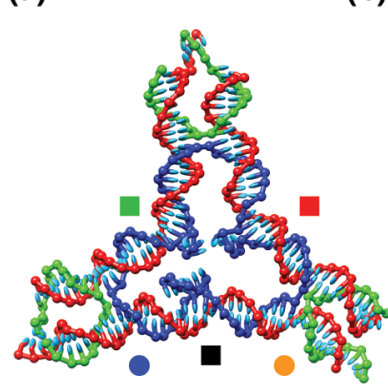

4-bulge

7-bulge

4-bulge

4-way junction 1

4-way junction 2

(b)
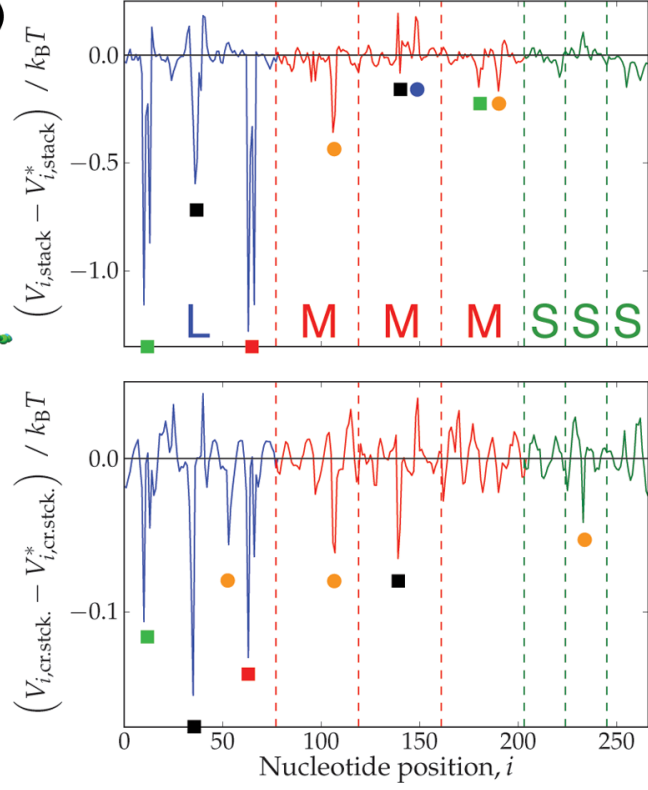
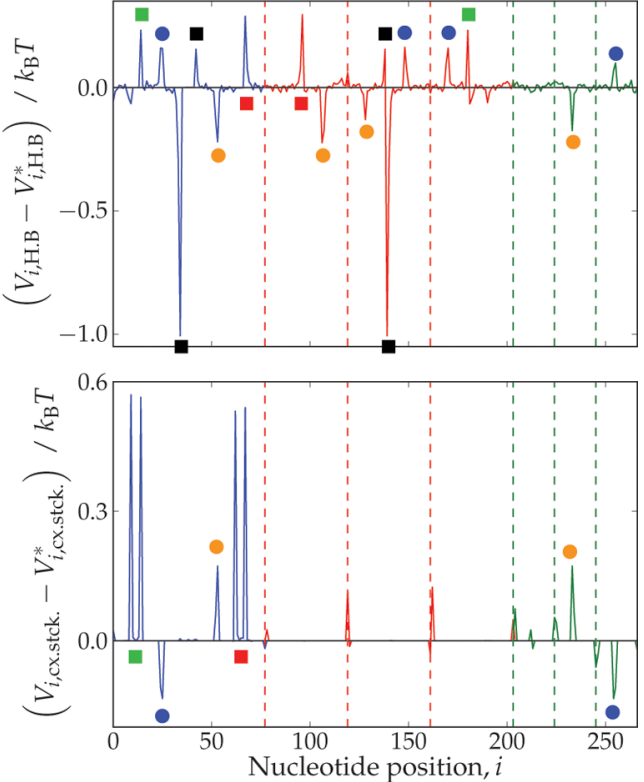

Figure 10. (a) Illustration of a 744 tile configuration. (b) The contributions to the difference in potential energy between the two prism forms for the $i$ th nucleotide in a tile are plotted for different terms in the oxDNA potential. The relative locations of the $L, M$, and $S$ strands, which are color coordinated with the schematic picture of a 744 tile in (a), are listed. Note that the energies were averaged over the six tiles in a nanoprism. In (a) and (b) circles denote base(s) that are in the vicinity of or located at a four-way junction. Similarly, squares denotes base(s) that are near or part of a bulge. Circles and squares are drawn only when the absolute magnitude of the energy difference is $\geq 0.05 k_{\mathrm{B}} T$.

Table 1. Difference in the Potential Energy Between the 744 and r744 Prisms at $20^{\circ} \mathrm{C}$ Broken down into the Contributions from Different Terms in the oxDNA Potential

\begin{tabular}{lr}
\multicolumn{1}{c}{ interaction type } & $\Delta V / k_{\mathrm{B}} T$ \\
stacking, $V_{\text {stack }}$ & -64.6 \\
hydrogen bonding, $V_{\mathrm{HB}}$ & -7.9 \\
cross-stacking, $V_{\text {cr.stck. }}$ & -1.0 \\
coaxial-stacking, $V_{\text {cx.stck. }}$ & 15.8 \\
total potential energy, $V$ & -55.6
\end{tabular}

The complexities of large DNA nanostructures are such that simple geometric arguments and rules of thumb are likely to be insufficient for the rational design of ever more complex structures.

\section{CONCLUSIONS}

Here, we have used the oxDNA coarse-grained model and molecular simulations to characterize the structural properties of exemplar large DNA polyhedral nanostructures in unprecedented detail. One important aspect of our results is that they showcase oxDNA's ability to reproduce the relevant experimental results, in this case the overall structure of the prisms as revealed by cryoTEM, remarkably well. In particular, first we are able to reproduce the variation of the chiral twist of the top and bottom faces as the sizes of the bulges (in the centers of the tiles) are varied, even including the slightly nonzero average twist for the "symmetric" 744 tiles. Second, we are able to reproduce the preference for the experimentally observed structure rather than its inverted isomer. This reproduction of, in some cases, quite detailed and subtle features gives us confidence in the model's utility for structural prediction of DNA nanostructures.

But more than this, our simulations are also able to provide information that is not straightforwardly available from experiment. First, our simulations not only provide a picture of the average structure of the prisms but also allow a statistical characterization of the fluctuations that are possible. Although the prisms have well-defined structures, there is still significant flexibility as evidenced by the fluctuations in the relative orientations (i.e., twisting) and position (i.e., shearing) of the top and bottom triangular faces.

Second, the oxDNA model can provide greater physical insight into the underlying causes of the observed behavior. For example, although it is perhaps unsurprising that bulges with more nucleotides are more likely to lead to larger bend angles (and hence can be used to control the chiral twist of the prisms), our simulations reveal that this behavior arises from a combination of two effects. First, bulged duplexes with smaller bulges prefer to adopt a straighter "stacked" geometry where the stacking opposite the bulge is maintained thus allowing only modest angular deviations in the duplex. Second, the more hinge-like "unstacked" state of the bulged duplex is able to open up to larger bend angles when the connecting bulge loop is longer.

Third, our simulations allow us to characterize how the structure of the components, in this case the three-arm tiles, evolves as the target structure is assembled. In particular, we found that the individual tiles can adopt a wide diversity of structures facilitated both by the flexibility due to the bulge loops and the relatively mild additional constraint provided by the single four-way junction in each arm on the relative orientations of the two helices that make up the arm. However, in the prisms the tiles have a much more well-defined and rigid structure because of the additional constraints provided both by the individual interarm bonds, which make the prism edges stiff and straight, and by the overall structure of the prism, which restricts the relative orientations of the arms.

Furthermore, we also found that certain properties of the tiles only became evident once assembled. In particular, the individual tiles are, on average, basically flat, with a preferred curvature only appearing and becoming more pronounced as 
the polygonal faces of the prism are formed. This developing curvature means that the self-assembly pathway will naturally lead to the formation of the experimentally observed form of the prism rather than its inverted isomer.

Our simulations also help us to deduce more general principles about the structure of DNA polyhedra. First, we have provided mechanistic insights into the empirically deduced design rules for multi-arm star tiles, namely the effect of bulge size on average bend angle, and the preference for tiles to assemble with a preferred curvature. Second, for a system where the number of polyhedral edges $N_{\mathrm{e}}$ is less than the degrees of freedom associated with the polyhedral vertices $\left(3 N_{\mathrm{v}}-6\right.$, where $N_{\mathrm{v}}$ is the number of vertices), significant flexibility in the polyhedron should be expected, because the star tiles only provide a mild constraint on the bend angle at the vertices. For the current case, $3 N_{\mathrm{v}}-6=12$, whereas $N_{\mathrm{e}}=9$ and the three remaining unconstrained degrees of freedom correspond to the twisting and shearing modes of deformation. For fully triangulated DNA polyhedra, such as the tetrahedron, ${ }^{22}$ octahedron $^{43}$ and icosahedron, ${ }^{23}$ where $3 N_{\mathrm{v}}-6=N_{\mathrm{e}}$, the structures are expected to be much more rigid, whereas structures like the dodecahedron ${ }^{22}\left(3 N_{\mathrm{v}}-6=54\right.$ and $\left.N_{\mathrm{e}}=30\right)$ are expected to be very flexible. The origami tripod tiles provide an interesting contrast to the star tiles considered here, as struts between the arms constrain the interarm angles, both rigidifying the tiles and the resulting polyhedra, as well as allowing a variety of polyhedral nanoprisms to be assembled by varying the interarm angles. ${ }^{44}$

The DNA multi-arm tiles considered here can be considered as an example of a "patchy" particle. The synthesis of "patchy particles" has been a particular recent focus for the colloidal and nanoparticle community in order to increase the range of structures that can be created, ${ }^{65,66}$ and there have also been many simulations studies exploring the finite and extended structures that could be created from such patchy particles. ${ }^{67,68}$ Interestingly, there is considerable overlap between the structures experimentally observed for the multi-arm DNA tiles and those obtained in simulations of patchy particles with the same numbers of patches as arms in the tiles. ${ }^{69-71}$ However, so far the structures formed by the tiles have been restricted to $2 \mathrm{D}$ arrays $^{40}$ and polyhedral shells. ${ }^{22,23,43,44,72}$ To obtain 3D extended structures, such as the diamond lattices observed in simulations of four-patch particles, ${ }^{73,74}$ would require greater control of the $3 \mathrm{D}$ arrangement of the tile arms than is possible in the flexible star-tile motifs, but may be possible in four-arm analogues of the origami tripod tiles.

Overall, our results illustrate the power of coarse-grained modeling and of oxDNA, in particular, to characterize large DNA nanostructures. This success stems both from the model's ability to accurately capture the relevant biophysical properties of DNA and from its computational efficiency. Combined with the parallelization available through GPU computing, such studies have the potential to become routine, in a way that would be impossible for all-atom models for the foreseeable future. For example, we are currently applying the model to characterize a wide range of DNA origamis.

We also believe that the physical insights available from oxDNA simulations can play an important role in the rational design of future DNA nanostructures. Furthermore, the predictive power of the model could be used to prescreen potential designs to test whether they exhibit the required properties prior to experimental realization.
The oxDNA model can also be used to go beyond structural characterization to study the self-assembly mechanisms of DNA nanostructures. For example, for the current nanoprisms one might ask why triangular rather than square (or for that matter pentagonal) prisms are the dominant product when all would be maximally base paired. To address this question we are currently studying how the relative flexibility of tiles induced by different bulge sizes controls the rates of closure of trimers of tiles to form triangular faces.

\section{METHODS}

We ran long molecular dynamics (MD) simulations to generate representative sets of configurations for each star tile and nanoprism considered. Typical total simulation lengths for both tile and prism systems were at least $5 \times 10^{9}$ simulation steps. Each set consisted of thousands of configurations, where the total energy and the bend angles for the bulged duplexes were decorrelated from that of any other configuration in the set. The sets of configurations are then used to measure the equilibrium structural properties reported in earlier sections. Errors for the quantities measured were estimated by computing the standard error of the mean value from multiple independent simulations for each system. In most cases, the error bars in the figures are smaller than the point sizes.

In the MD simulations, an Andersen-like thermostat was used, which generates diffusive motion of particles beyond an extremely short time scale. ${ }^{75}$ Running the simulations on GPUs rather than CPUs speeds up typical MD simulations by roughly a factor of 25 . Additionally, all simulations were performed at $T_{\text {sim }}=20-25{ }^{\circ} \mathrm{C}$, the temperature range from which the cryoTEM quenching likely took place, and with $\left[\mathrm{Na}^{+}\right]=0.5 \mathrm{M}$. The experiments were also performed in this high-salt regime, where the electrostatic repulsion between charged nucleotides is highly screened by the ionic solution. Further details pertaining to the oxDNA model can be found in ref 48, while additional information regarding the simulations performed in this article can be found in the Supporting Information.

\section{ASSOCIATED CONTENT}

\section{S Supporting Information}

The Supporting Information is available free of charge on the ACS Publications website at DOI: 10.1021/acsnano.5b07664.

Outlines of the different schemes used to measure various bending and twisting angles, as well as the degree of prism shearing, is presented. Additional simulation results are also presented and discussed (PDF).

W Web-Enhanced Features

Animations of a tile and a prism.

\section{AUTHOR INFORMATION}

\section{Corresponding Authors}

*E-mail: john.schreck@chem.ox.ac.uk.

*E-mail: jonathan.doye@chem.ox.ac.uk.

\section{Notes}

The authors declare no competing financial interest.

\section{ACKNOWLEDGMENTS}

The authors are grateful to the Engineering and Physical Sciences Research Council for financial support [EP/J019445/ 1] and acknowledge the use of the University of Oxford Advanced Research Computing (ARC) facility in carrying out this work, http://dx.doi.org/10.5281/zenodo.22558.

\section{REFERENCES}

(1) Hamblin, G. D.; Carneiro, K. M.; Fakhoury, J. F.; Bujold, K. E.; Sleiman, H. F. Rolling Circle Amplification-Templated DNA Nano- 
tubes Show Increased Stability and Cell Penetration Ability. J. Am. Chem. Soc. 2012, 134, 2888-2891.

(2) Wollman, A. J.; Sanchez-Cano, C.; Carstairs, H. M.; Cross, R. A.; Turberfield, A. J. Transport and Self-Organization Across Different Length Scales Powered by Motor Proteins and Programmed by DNA. Nat. Nanotechnol. 2014, 9, 44-47.

(3) Goodman, R. P.; Heilemann, M.; Doose, S.; Erben, C. M.; Kapanidis, A. N.; Turberfield, A. J. Reconfigurable, Braced, ThreeDimensional DNA Nanostructures. Nat. Nanotechnol. 2008, 3, 93-96.

(4) Adleman, L. M. Molecular Computation of Solutions to Combinatorial Problems. Science 1994, 266, 1021-1024.

(5) Seeman, N. C. Nucleic Acid Junctions and Lattices. J. Theor. Biol. 1982, 99, 237-247.

(6) Rothemund, P. W. K. Folding DNA to Create Nanoscale Shapes and Patterns. Nature 2006, 440, 297-302.

(7) Douglas, S. M.; Dietz, H.; Liedl, T.; Högberg, B.; Graf, F.; Shih, W. M. Self-Assembly of DNA into Nanoscale Three-Dimensional Shapes. Nature 2009, 459, 414-418.

(8) Liedl, T.; Högberg, B.; Tytell, J.; Ingber, D. E.; Shih, W. M. SelfAssembly of Three-Dimensional Prestressed Tensegrity Structures from DNA. Nat. Nanotechnol. 2010, 5, 520-524.

(9) Ke, Y.; Ong, L. L.; Shih, W. M.; Yin, P. Three-Dimensional Structures Self-Assembled from DNA Bricks. Science 2012, 338, 11771183

(10) Wei, B.; Dai, M.; Yin, P. Complex Shapes Self-Assembled from Single-Stranded DNA Tiles. Nature 2012, 485, 623-626.

(11) Han, D.; Pal, S.; Yang, Y.; Jiang, S.; Nangreave, J.; Liu, Y.; Yan, H. DNA Gridiron Nanostructures Based on Four-Arm Junctions. Science 2013, 339, 1412-1415.

(12) Iinuma, R.; Ke, Y.; Jungmann, R.; Schlichthaerle, T.; Woehrstein, J. B.; Yin, P. Polyhedra Self-Assembled from DNA Tripods and Characterized with 3D DNA-PAINT. Science 2014, 344, 65-69.

(13) Gerling, T.; Wagenbauer, K. F.; Neuner, A. M.; Dietz, H. Dynamic DNA Devices and Assemblies Formed by ShapeComplementary, Non-Base Pairing 3D Components. Science 2015, 347, 1446-1452.

(14) Cademartiri, L.; Bishop, K. J. M. Programmable Self-Assembly. Nat. Mater. 2015, 14, 2-9.

(15) Douglas, S. M.; Marblestone, A. H.; Teerapittayanon, S.; Vazquez, A.; Church, G. M.; Shih, W. M. Rapid Prototyping of 3D DNA-Origami Shapes with caDNAno. Nucleic Acids Res. 2009, 37, 5001-5006.

(16) Benson, E.; Mohammed, A.; Gardell, J.; Masich, S.; Czeizler, E.; Orponen, P.; Högberg, B. DNA Rendering of Polyhedral Meshes at the Nanoscale. Nature 2015, 523, 441-444.

(17) Winfree, E.; Liu, F. R.; Wenzler, L. A.; Seeman, N. C. Design and Self-Assembly of Two-Dimensional DNA Crystals. Nature 1998, 394, 539.

(18) Zheng, J.; Birktoft, J. J.; Chen, Y.; Wang, T.; Sha, R.; Constantinou, P. E.; Ginell, S. L.; Mao, C.; Seeman, N. C. From Molecular to Macroscopic via the Rational Design of a Self-Assembled 3D DNA Crystal. Nature 2009, 461, 74-77.

(19) Zhao, J.; Chandrasekaran, A. R.; Li, Q.; Li, X.; Sha, R.; Seeman, N. C.; Mao, C. Post-Assembly Stabilization of Rationally Designed DNA Crystals. Angew. Chem., Int. Ed. 2015, 54, 9936-9939.

(20) Shih, W. M.; Quispe, J. D.; Joyce, G. F. A 1.7-Kilobase SingleStranded DNA that Folds into a Nanoscale Octahedron. Nature 2004, $427,618-621$.

(21) Goodman, R. P.; Schaap, I. A.; Tardin, C. F.; Erben, C. M.; Berry, R. M.; Schmidt, C. F.; Turberfield, A. J. Rapid Chiral Assembly of Rigid DNA Building Blocks for Molecular Nanofabrication. Science 2005, 310, 1661-1665.

(22) He, Y.; Ye, T.; Su, M.; Zhang, C.; Ribbe, A.; Jiang, W.; Mao, C. Hierarchical Self-Assembly of DNA into Symmetric Supramolecular Polyhedra. Nature 2008, 452, 198-201.

(23) Zhang, C.; Su, M.; He, Y.; Zhao, X.; Fang, P.-a.; Ribbe, A. E.; Jiang, W.; Mao, C. Conformational Flexibility Facilitates Self-Assembly of Complex DNA Nanostructures. Proc. Natl. Acad. Sci. U. S. A. 2008, 105, 10665-10669.

(24) Zhang, C.; Ko, S. H.; Su, M.; Leng, Y.; Ribbe, A. E.; Jiang, W.; Mao, C. Symmetry Controls the Face Geometry of DNA Polyhedra. J. Am. Chem. Soc. 2009, 131, 1413-1415.

(25) Kočar, V.; Schreck, J. S.; Čeru, S.; Gradivsar, H.; Bašić, N.; Pisanski, T.; Doye, J. P. K.; Jerala, R. Design principles for rapid folding of knotted DNA nanostructures. Nat. Commun. 2016, 7, 10803.

(26) Simmel, S. S.; Nickels, P. C.; Liedl, T. Wireframe and Tensegrity DNA Nanostructures. Acc. Chem. Res. 2014, 47, 1691-1699.

(27) Zhang, F.; Jiang, S.; Wu, S.; Li, Y.; Mao, C.; Liu, Y.; Yan, H. Complex Wireframe DNA Origami Nanostructures with Multi-Arm Junction Vertices. Nat. Nanotechnol. 2015, 10, 779-784.

(28) Han, D.; Pal, S.; Liu, Y.; Yan, H. Folding and Cutting DNA into Reconfigurable Topological Nanostructures. Nat. Nanotechnol. 2010, $5,712-717$.

(29) Green, S.; Bath, J.; Turberfield, A. Coordinated Chemomechanical Cycles: A Mechanism for Autonomous Molecular Motion. Phys. Rev. Lett. 2008, 101, 238101.

(30) Tomov, T. E.; Tsukanov, R.; Liber, M.; Masoud, R.; Plavner, N.; Nir, E. Rational Design of DNA Motors: Fuel Optimization Through Single-Molecule Fluorescence. J. Am. Chem. Soc. 2013, 135, 1193511941.

(31) Sobczak, J.-P. J.; Martin, T. G.; Gerling, T.; Dietz, H. Rapid Folding of DNA Into Nanoscale Shapes at Constant Temperature. Science 2012, 338, 1458-1461.

(32) Castro, C. E.; Kilchherr, F.; Kim, D.-N.; Shiao, E. L.; Wauer, T.; Wortmann, P.; Bathe, M.; Dietz, H. A Primer to Scaffolded DNA Origami. Nat. Methods 2011, 8, 221-229.

(33) Kim, D.-N.; Kilchherr, F.; Dietz, H.; Bathe, M. Quantitative Prediction of 3D Solution Shape and Flexibility of Nucleic Acid Nanostructures. Nucleic Acids Res. 2012, 40, 2862-2868.

(34) Marras, A. E.; Zhou, L.; Su, H.-J.; Castro, C. E. Programmable Motion of DNA Origami Mechanisms. Proc. Natl. Acad. Sci. U. S. A. 2015, 112, 713-718.

(35) Dietz, H.; Douglas, S. M.; Shih, W. M. Folding DNA Into Twisted and Curved Nanoscale Shapes. Science 2009, 325, 725-730.

(36) Lin, C.; Liu, Y.; Rinker, S.; Yan, H. DNA Tile Based SelfAssembly: Building Complex Nanoarchitectures. ChemPhysChem 2006, 7, 1641-1647.

(37) Rangnekar, A.; LaBean, T. H. Tile-Based DNA NanoAssemblies. Nucleic Acid Nanotechnology; Springer-Verlag: Berlin, 2014; pp 71-92.

(38) Fu, T. J.; Seeman, N. C. DNA Double-Crossover Molecules. Biochemistry 1993, 32, 3211-3220.

(39) LaBean, T. H.; Yan, H.; Kopatsch, J.; Liu, F.; Winfree, E.; Reif, J. H.; Seeman, N. C. Construction, Analysis, Ligation, and Self-Assembly of DNA Triple Crossover Complexes. J. Am. Chem. Soc. 2000, 122, $1848-1860$

(40) Yan, H.; Park, S.; Finkelstein, G.; Reif, J. H.; LaBean, T. H. DNA-Templated Self-Assembly of Protein Arrays and Highly Conductive Nanowires. Science 2003, 301, 1882-1884.

(41) Zhang, C.; He, Y.; Su, M.; Ko, S. H.; Ye, T.; Leng, Y.; Sun, X.; Ribbe, A. E.; Jiang, W.; Mao, C. DNA Self-Assembly: From 2D to 3D. Faraday Discuss. 2009, 143, 221-233.

(42) Sa-Ardyen, P.; Vologodskii, A. V.; Seeman, N. C. The Flexibility of DNA Double Crossover Molecules. Biophys. J. 2003, 84, 38293837.

(43) He, Y.; Su, M.; Fang, P.-a.; Zhang, C.; Ribbe, A. E.; Jiang, W.; Mao, C. On the Chirality of Self-Assembled DNA Octahedra. Angew. Chem. 2010, 122, 760-763.

(44) Zhang, C.; Wu, W.; Li, X.; Tian, C.; Qian, H.; Wang, G.; Jiang, W.; Mao, C. Controlling the Chirality of DNA Nanocages. Angew. Chem., Int. Ed. 2012, 51, 7999-8002.

(45) Tian, C.; Li, X.; Liu, Z.; Jiang, W.; Wang, G.; Mao, C. Directed Self-Assembly of DNA Tiles into Complex Nanocages. Angew. Chem. 2014, 126, 8179-8182. 
(46) Liu, Z.; Tian, C.; Yu, J.; Li, Y.; Jiang, W.; Mao, C. Self-Assembly of Responsive Multilayered DNA Nanocages. J. Am. Chem. Soc. 2015, 137, 1730-1733.

(47) Li, Y.; Tian, C.; Liu, Z.; Jiang, W.; Mao, C. Structural Transformation: Assembly of an Otherwise Inaccessible DNA Nanocage. Angew. Chem., Int. Ed. 2015, 54, 5990-5993.

(48) Snodin, B. E.; Randisi, F.; Mosayebi, M.; Šulc, P.; Schreck, J. S.; Romano, F.; Ouldridge, T. E.; Tsukanov, R.; Nir, E.; Louis, A. A.; et al. Introducing Improved Structural Properties and Salt Dependence into a Coarse-Grained Model of DNA. J. Chem. Phys. 2015, 142, 234901.

(49) He, Y.; Chen, Y.; Liu, H.; Ribbe, A. E.; Mao, C. Self-Assembly of Hexagonal DNA Two-Dimensional (2D) Arrays. J. Am. Chem. Soc. 2005, 127, 12202-12203.

(50) Falconi, M.; Oteri, F.; Chillemi, G.; Andersen, F. F.; Tordrup, D.; Oliveira, C. L.; Pedersen, J. S.; Knudsen, B. R.; Desideri, A. Deciphering the Structural Properties that Confer Stability to a DNA Nanocage. ACS Nano 2009, 3, 1813-1822.

(51) Oteri, F.; Falconi, M.; Chillemi, G.; Andersen, F. F.; Oliveira, C. L.; Pedersen, J. S.; Knudsen, B. R.; Desideri, A. Simulative Analysis of a Truncated Octahedral DNA Nanocage Family Indicates the SingleStranded Thymidine Linkers as the Major Player for the Conformational Variability. J. Phys. Chem. C 2011, 115, 16819-16827.

(52) Yoo, J.; Aksimentiev, A. In Situ Structure and Dynamics of DNA Origami Determined Through Molecular Dynamics Simulations. Proc. Natl. Acad. Sci. U. S. A. 2013, 110, 20099-20104.

(53) Iacovelli, F.; Alves, C.; Falconi, M.; Oteri, F.; Oliveira, C. L.; Desideri, A. Influence of the Single-Strand Linker Composition on the Structural/Dynamical Properties of a Truncated Octahedral DNA Nano-Cage Family. Biopolymers 2014, 101, 992-999.

(54) Srinivas, N.; Ouldridge, T. E.; Sulc, P.; Schaeffer, J.; Yurke, B.; Louis, A. A.; Doye, J. P. K.; Winfree, E. On the Biophysics and Kinetics of Toehold-Mediated DNA Strand Displacement. Nucleic Acids Res. 2013, 41, 10641-10658.

(55) Ouldridge, T. E.; Šulc, P.; Romano, F.; Doye, J. P. K.; Louis, A. A. DNA Hybridization Kinetics: Zippering, Internal Displacement and Sequence Dependence. Nucleic Acids Res. 2013, 41, 8886-8895.

(56) Schreck, J. S.; Ouldridge, T. E.; Romano, F.; Šulc, P.; Shaw, L. P.; Louis, A. A.; Doye, J. P. K. DNA Hairpins Destabilize Duplexes Primarily by Promoting Melting Rather than by Inhibiting Hybridization. Nucleic Acids Res. 2015, 43, 6181-6190.

(57) Mosayebi, M.; Louis, A. A.; Doye, J. P. K.; Ouldridge, T. E. Force-Induced Rupture of a DNA Duplex. ACS Nano 2015, 9, 1199312003.

(58) Doye, J. P. K.; Ouldridge, T. E.; Louis, A. A.; Romano, F.; Šulc, P.; Matek, C.; Snodin, B.; Rovigatti, L.; Schreck, J. S.; Harrison, R. M.; et al. Coarse-Graining DNA for Simulations of DNA Nanotechnology. Phys. Chem. Chem. Phys. 2013, 15, 20395-20414.

(59) Ouldridge, T. E.; Louis, A. A.; Doye, J. P. K. Structural, Mechanical and Thermodynamic Properties of a Coarse-Grained Model of DNA. J. Chem. Phys. 2011, 134, 085101.

(60) Ouldridge, T. E. Coarse-Grained Modelling of DNA and DNA Nanotechnology. Ph.D. Thesis, University of Oxford, 2011.

(61) Šulc, P.; Romano, F.; Ouldridge, T. E.; Rovigatti, L.; Doye, J. P. K.; Louis, A. A. Sequence-Dependent Thermodynamics of a CoarseGrained DNA Model. J. Chem. Phys. 2012, 137, 135101.

(62) Schreck, J. S.; Ouldridge, T. E.; Romano, F.; Louis, A. A.; Doye, J. P. K. Characterizing the Bending and Flexibility Induced by Bulges in DNA Duplexes. J. Chem. Phys. 2015, 142, 165101.

(63) oxDNA, https://dna.physics.ox.ac.uk (accessed March 23, 2016).

(64) Lilley, D. M. J. Structures of Helical Junctions in Nucleic Acids. Q. Rev. Biophys. 2000, 33, 109-159.

(65) Glotzer, S. C.; Solomon, M. Anisotropy of Building Blocks and Their Assembly Into Complex Structures. Nat. Mater. 2007, 6, 557562.

(66) Wang, Y.; Wang, Y.; Breed, D. R.; Manoharan, V. N.; Feng, L.; Hollingsworth, A. D.; Weck, M.; Pine, D. J. Colloids With Valence and Specific Directional Bonding. Nature 2012, 491, 51-55.
(67) Zhang, Z.; Glotzer, S. C. Self-Assembly of Patchy Particles. Nano Lett. 2004, 4, 1407-1413.

(68) Bianchi, E.; Blaak, R.; Likos, C. N. Patchy Colloids: State of the Art and Perspectives. Phys. Chem. Chem. Phys. 2011, 13, 6397-6410.

(69) Doye, J. P. K.; Louis, A. A.; Lin, I.-C.; Allen, L. R.; Noya, E. G.; Wilber, A. W.; Kok, H. C.; Lyus, R. Controlling Crystallization and its Absence: Proteins, Colloids and Patchy Models. Phys. Chem. Chem. Phys. 2007, 9, 2197-2205.

(70) Wilber, A. W.; Doye, J. P. K.; Louis, A. A.; Lewis, A. C. F. Monodisperse Self-Assembly in a Model with Protein-like Interactions. J. Chem. Phys. 2009, 131, 175102.

(71) van der Linden, M. N.; Doye, J. P. K.; Louis, A. A. Formation of Dodecagonal Quasicrystals in Two-Dimensional Systems of Patchy Particles. J. Chem. Phys. 2012, 136, 054904.

(72) Zhang, F.; Liu, Y.; Yan, H. Complex Archimedean Tiling SelfAssembled from DNA Nanostructures. J. Am. Chem. Soc. 2013, 135, $7458-7461$.

(73) Zhang, Z.; Keys, A. S.; Chen, T.; Glotzer, S. C. Self-Assembly of Patchy Particles Into Diamond Structures Through Molecular Mimicry. Langmuir 2005, 21, 11547-11551.

(74) Romano, F.; Sanz, E.; Sciortino, F. Crystallization of Tetrahedral Patchy Particles In Silico. J. Chem. Phys. 2011, 134, 174502.

(75) Russo, J.; Tartaglia, P.; Sciortino, F. Reversible Gels of Patchy Particles: Role of the Valence. J. Chem. Phys. 2009, 131, 014504. 